\title{
Use of central and peripheral temperature measure- ments in care of the critically ill child
}

\author{
ALBERT AYNSLEY-GREEN and DOUGLAS PICKERING \\ From the Department of Paediatrics, The Radcliffe Infirmary, Oxford
}

\begin{abstract}
Aynsley-Green, A., and Pickering, D. (1974). Archives of Disease in Childhood, 49,477. Use of central and peripheral temperature measurements in care of the critically ill child. In the presence of constant ambient conditions, a fall in effective circulating blood volume causes peripheral vasoconstriction which is reflected in a fall in peripheral (great toe) temperature and thus in an increase in the central (rectal)/peripheral temperature gradient. We report 5 illustrative clinical situations showing the value of this technique in the medical care of critically ill children. We suggest that such measurements are useful, first in the recognition of dehydration and in assessing the response to treatment, secondly in managing shock, and finally, in differentiating dehydration fever from other causes of fever.
\end{abstract}

A qualitative assessment of peripheral vasoconstriction can be obtained by measuring the gradient between central (rectal) and peripheral (great toe) temperature. Ibsen (1967) pointed out that in the presence of a constant environmental temperature, a fall in effective circulating blood volume causes peripheral vasoconstriction and hence an increase in the central/peripheral temperature gradient. He confirmed this by withdrawing 500 $\mathrm{ml}$ blood from an adult subject and showing that a fall in peripheral skin temperature of up to $8{ }^{\circ} \mathrm{C}$ followed. The normal gradient was re-established when the blood volume was replaced. Sequential measurements of the rectal and great toe temperature have been shown to be valuable in managing postoperative hypovolaemic shock and hypovolaemia complicated by diminished cardiac function in adults (Ross, Brock, and Aynsley-Green, 1969; Pagliero, Yates, and Hill, 1973).

We have measured rectal and great toe temperature gradients in seriously ill children to determine whether such simple noninvasive bedside observations would be useful in assessing the state of hydration and the effective circulating blood volume. We report 5 situations which occurred during the management of 4 children where temperature recordings considerably helped in deciding the most appropriate treatment for the child.

Received 28 November 1973.
The children had routine recordings of pulse rate, blood pressure, and urine output. Central and peripheral temperatures were recorded with thermistors and an electric thermometer (Yellow Springs Instrument Co., Ohio, U.S.A.) by means of a rectal probe inserted 6 to $8 \mathrm{~cm}$ into the rectum and a surface applicator attached to the lateral side of the great toe. Room temperature was constant during each period of observation, the range being between 24 and $26^{\circ} \mathrm{C}$.

\section{Case reports}

Case 1. An 18-month-old child had severe viral pneumonia and required positive pressure ventilation. 24 hours after admission she developed an unexplained tachycardia with peripheral vasoconstriction. Measurement of central and peripheral temperatures showed a gradient of $11{ }^{\circ} \mathrm{C}$ (Fig. 1). We decided that these observations indicated a low effective circulating blood volume, despite maintenance of normal blood pressure. This conclusion was supported by showing a raised packed cell volume of $48 \%$. Accordingly, intravenous and oral fluids were rapidly given. Within 3 hours and after the administration of $200 \mathrm{ml}$ fluid, the peripheral temperature approximated to the central temperature and the tachycardia resolved. Improvement in peripheral tissue perfusion was reflected by an increase in urine output.

Case 2. A 4-year-old girl was admitted in diabetic ketoacidosis. Clinical examination revealed tachycardia 


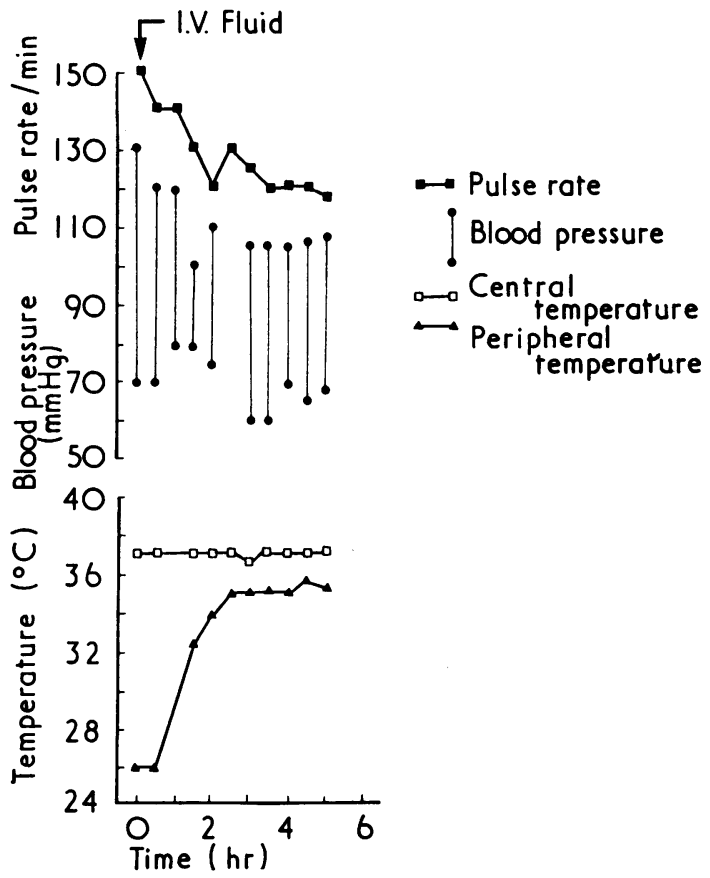

FIG. 1.-Case 1. Mild dehydration. Peripheral vasoconstriction is reflected in a wide temperature gradient. Administration of fluid caused a progressive approximation of peripheral and central temperatures and a fall in pulse rate.

and peripheral vasoconstriction with both a low central and peripheral temperature (Fig. 2). The initial blood glucose level was $710 \mathrm{mg} / 100 \mathrm{ml}$. Rapid fluid administration, including $25 \mathrm{ml}$ of $4 \cdot 2 \%$ sodium bicarbonate, improved the air hunger and reduced the blood glucose level to $480 \mathrm{mg} / 100 \mathrm{ml}$. This was accompanied by a rise in both temperature recordings. Two injections of 2 units insulin intramuscularly, after 4 and 7 hours, caused a marked improvement in the child's general condition, but the persistence of the wide central/peripheral temperature gradient suggested continuing dehydration.

Fluid replacement therapy continued even though the child's general condition had improved sufficiently to be able to take oral fluid. It was only after 14 hours that the temperature gradient returned to normal. This then clearly indicated that fluid replacement was no longer necessary. Over $2 \cdot 01$. fluid had been given by this time.

Case 3. The same child reported in Case 1 later developed, after 10 days of positive pressure ventilation, an acute collapse associated with an Esch. coli pneumonia. At this time she had tachycardia, subnormal temperature, and peripheral vasoconstriction (Fig. 3). She was given intravenous hydrocortisone, cloxacillin, and gentamicin, and a blood transfusion was started. She was wrapped

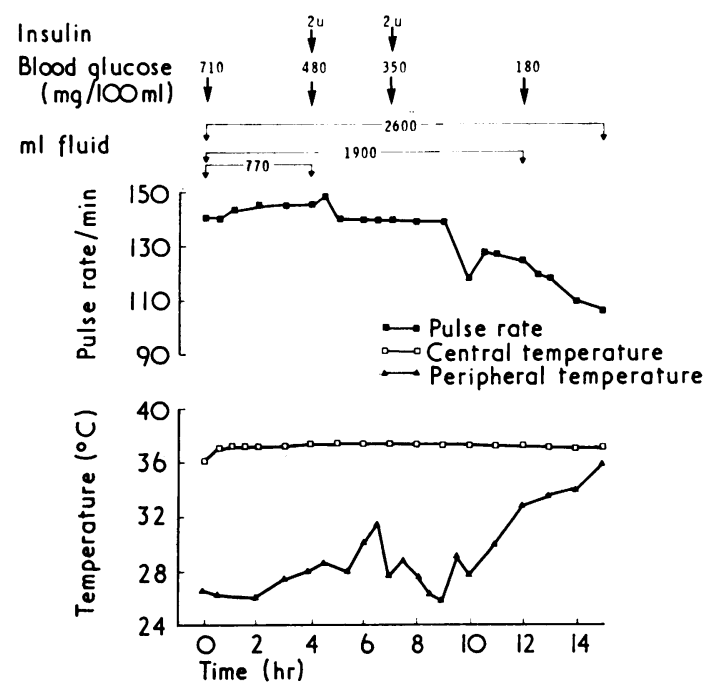

Fig. 2.-Case 2. Dehydration in diabetic ketoacidosis. Temperature measurements allowed the response to rehydration to be monitored and an end-point for this therapy to be recognized. Cumulative totals of intravenous and oral fluids are shown.

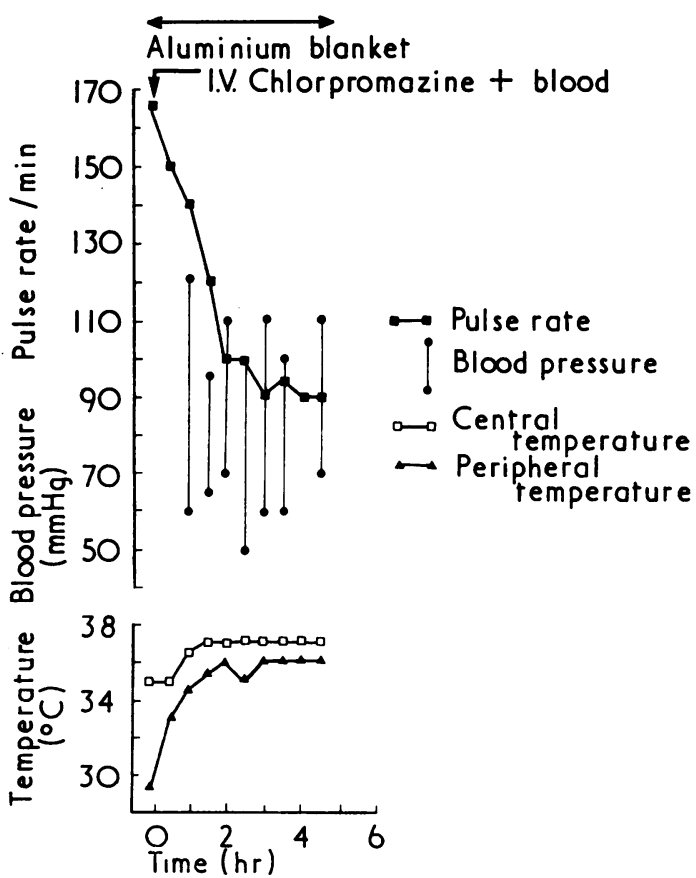

Fig. 3.-Case 3. Septicaemic shock. A rapid blood transfusion combined with administration of chlorpromazine caused the restoration of normal skin perfusion and fall in tachycardia. 


\section{Use of central and peripheral temperature measurements in care of the critically ill child}

in aluminium foil and $5 \mathrm{mg}$ intravenous chlorpromazine was given as a mild $\alpha$-adrenergic blocking drug to encourage peripheral vasodilatation. Blood was given quickly initially to prevent hypotension which could have followed sudden peripheral vasodilatation. Within $2 \frac{1}{2}$ hours and after the administration of $80 \mathrm{ml}$ blood, there was a marked improvement in her condition, the pulse rate fell, there was an increase in urine flow, and the normal temperature gradient was re-established. The child made an uneventful recovery.

Case 4. A 3-month-old child was admitted with pneumonia and high fever which had not responded to antibiotics or to treatment for 3 days with exposure to a cold current of air and sponging with tepid water. He had been vomiting and had refused his feeds during the illness. On admission the most striking features were tachycardia and intense peripheral vasoconstriction, which was confirmed by a temperature gradient of $12^{\circ} \mathrm{C}$ together with a central temperature of $39^{\circ} \mathrm{C}$ (Fig. 4). Our conclusion from these observations was that the child was dehydrated and this was confirmed by the initial laboratory results showing plasma osmolality 374 $\mathrm{mOsm} / \mathrm{kg}$ water, plasma sodium concentration 160 $\mathrm{mEq} / \mathrm{l}$., and blood urea level $120 \mathrm{mg} / 100 \mathrm{ml}$. The child was placed under an infant warmer with radiant heat applied, not to heat the child, but to provide a carefully controlled ambient temperature to encourage peripheral vasodilatation as rehydration was achieved.

During the next 16 hours, intragastric fluid was given
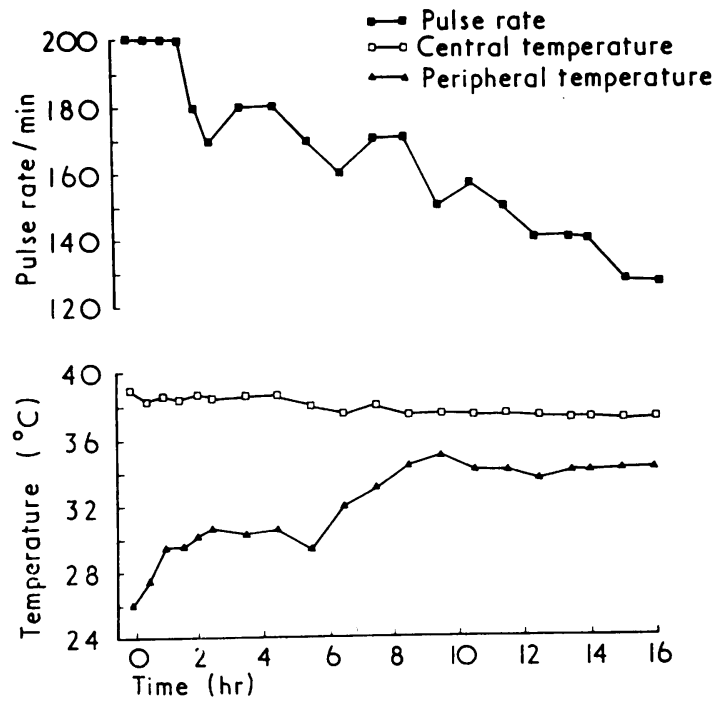

FIG. 4.-Case 4. Severe hypernatraemic dehydration with heat retention. Peripheral vasoconstriction due to dehydration is shown by the wide temperature gradient. Rehydration caused a progressive rise in peripheral temperature, restoration of a normal heat losing mechanism, and fall in pulse rate. by tube feed as $5 \%$ dextrose alternating with fullstrength milk formula at the rate of $50 \mathrm{ml} / \mathrm{hr}$. With this treatment there was a fall in pulse rate and restoration of a normal central temperature, together with approximation of the peripheral temperature. Plasma electrolytes and osmolality had returned to normal after 20 hours and the child was mentally alert without neurological sequelae.

Case 5. An 8-month-old infant was admitted for investigation of hydrocephalus. Inappropriate secretion of antidiuretic hormone was implied by showing a wide difference in the urine and plasma osmolality (387 and $254 \mathrm{mOsm} / \mathrm{kg}$ water, respectively), with a plasma sodium concentration of $116 \mathrm{mEq} / \mathrm{l}$. He was treated by restriction of water intake, and cautious administration of sodium. He became pyrexial after 1 week, with an increase in pulse rate. An increase in the temperature gradient suggested dehydration despite the persistence of low plasma sodium concentration $(126 \mathrm{mEq} / \mathrm{l}$.). Intravenous chlorpromazine $5 \mathrm{mg}$ was given, together with an infusion of $0.9 \%$ saline and dextrose saline. Within 8 hours of this treatment there was marked improvement in peripheral skin perfusion, restoration of normal central temperature, fall in pulse rate, and maintenance of satisfactory plasma electrolyte concentrations (Fig. 5).

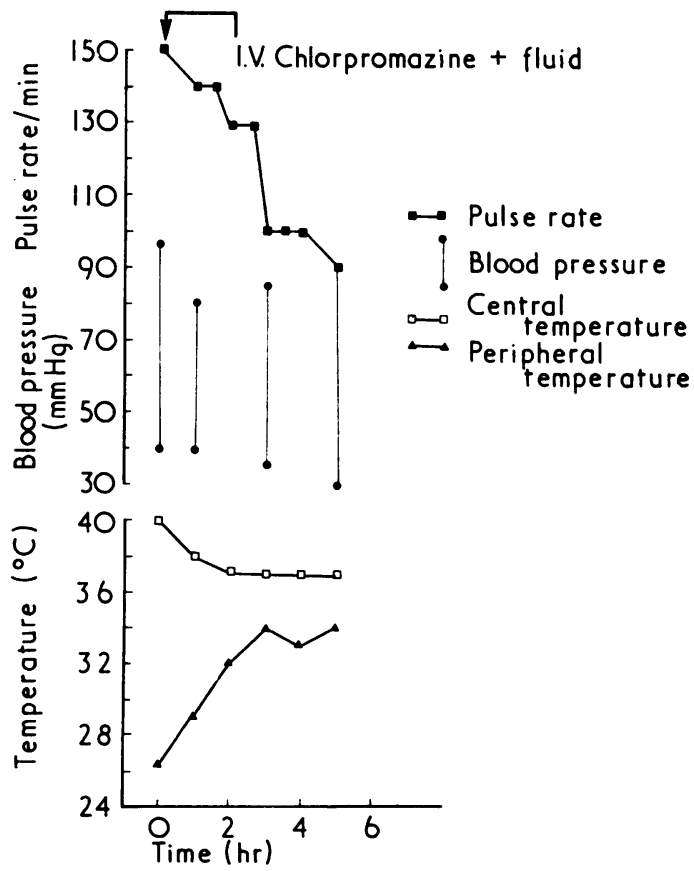

FIG. 5.-Case 5. Hyponatraemic dehydration with heat retention. Fluid replacement combined with chlorpromazine administration caused a rapid fall in central temperature, improvement in skin perfusion, and resolution of tachycardia. 


\section{Discussion}

The modern clinical thermometer was invented by Wunderlich in 1871 , and since then temperature recordings have become an accepted part of medical practice. However, on most occasions such recordings are made from one site alone, usually the mouth, axilla, or rectum. The prognostic significance of cold peripheries has been known since the time of Hippocrates (Adams, 1849), and it is well known that shocked patients are cold and vasoconstricted. Even with the development of thermistors and thermocouples, little attempt has been made to measure and record such peripheral temperature changes. Ibsen (1967) emphasized that in the presence of constant ambient conditions a fall in effective circulating blood volume causes peripheral vasoconstriction which is reflected in a decrease in peripheral temperature. Such bedside measurements have been shown to be useful in managing patients after cardiac surgery (Ross et al., 1969; Pagliero et al., 1973). Children are particularly susceptible to dehydration, and it is often difficult to assess both the fluid deficit and the response to treatment. Measurement of the temperature gradient in these patients, after excluding other causes of vasoconstriction, such as a cold environment, cardiac failure, or ergot poisoning, would theoretically be a useful clinical guide in assessing the effective circulating blood volume and the response to treatment.

We have presented observations obtained from 4 children in 5 clinical situations. The assessment of the temperature gradient within the clinical context of each situation considerably helped the management of the child.

Case 1 illustrates the fact that hypovolaemia can exist without clear clinical evidence of dehydration, and without hypotension. In the absence of heart failure and other causes of peripheral vasoconstriction, the increased temperature gradient was due to decreased effective circulating blood volume. This conclusion might have been reached by touch of the hand on the nose or the feet of the child, but the use of the electric thermometer allowed these changes to be accurately measured. Sequential recordings also allowed the response to treatment to be monitored.

Case 2 illustrates the value of temperature measurements in managing the dehydration of diabetic ketoacidosis. The improvement in peripheral temperature indicated when fluid replacement was no longer necessary: Ibsen states, -open up and fill up-stop when the feet get warm'!

A generally acceptable treatment for low cardiac output states after cardiac surgery in adults consists of the administration of $\alpha$-blocking drugs to overcome sympathetic overactivity, together with fluid transfusion to prevent subsequent hypotension (Dietzman et al., 1969). Such treatment causes peripheral vasodilatation and improvement in skin blood flow, and sequential measurements of central/ peripheral temperature gradients allow the response to treatment to be monitored (Ross et al., 1969).

Case 3 shows the application of this concept to treating septicaemic shock in a child. The use of $\alpha$-blocking drugs should be accompanied ideally by measurement of the central venous pressure to anticipate hypotension consequent to peripheral vasodilatation, but this is often technically not possible in children. The simultaneous and frequent recordings of pulse rate, blood pressure, and temperature measurements, however, allow this treatment to be safely employed.

Measurement of central and peripheral temperature gradients has shown the occurrence of hypovolaemic hyperpyrexia in adults (Ross et al., 1969; Spitzer and Brock, 1968). The fever in these patients is thought to be a consequence of hypovolaemia since the compensatory peripheral vasoconstriction prevents effective heat loss through the skin, and traditional treatment of the pyrexia by fanning in a cold current of air and sponging with tepid water aggravates the situation by causing further vasoconstriction. The condition is recognized by the presence of a wide temperature gradient, and logical treatment consists of fluid replacement with measures to encourage peripheral vasodilatation.

Cases 4 and 5 show the occurrence of this condition in children. It is our experience that in many children the fever accompanying an acute infective illness is aggravated by unrecognized dehydration, particularly when accompanied by vomiting, diarrhoea, and refusal to drink. We suggest that traditional treatment of fever by application of tepid water and exposure to a cold current of air is often inappropriate.

Thus, we have shown that in children the degree of peripheral vasoconstriction can be measured by recording central and peripheral temperature gradients. Vasoconstriction commonly accompanies states of dehydration and low effective circulating blood volume and these conditions respond to fluid replacement. Sequential temperature measurements, taken within the clinical context of each situation and after excluding other causes of vasoconstriction, are useful first in alerting one to the possibility of unrecognized dehydration, secondly in giving a simple, objective, noninvasive 


\section{Use of central and peripheral temperature measurements in care of the critically ill child 481}

bedside assessment of the response to treatment, thirdly in managing shock, and finally in recognizing dehydration fever. Formal measurements of blood volume were not carried out in the cases reported since we did not feel it was ethically justifiable to perform invasive and laborious investigations on sick children to substantiate bedside observations.

We are grateful to Dr. Hugh Ellis and Mr. Richard Gye for permission to publish details of Cases 2 and 5 , and to the nursing staff of the Special Care Unit, Leopold Surgical Ward and Leopold Medical Ward, for their invaluable assistance in recording parameters and in their care of the children. We also thank the Department of Medical Illustration for the figures.

\section{REFERENCES}

Adams, F. (1849). The Genuine Works of Hippocrates, Vol. 1, pp. 236, 242. Sydenham Society, London.
Dietzman, R. H., Ersek, R. A., Lillehei, C. W., Castaneda, A. R, and Lillehei, R. C. (1969). Low output syndrome. Recognition and treatment. Fournal of Thoracic and Cardiovascular Surgery, 57, 138.

Ibsen, B. (1967). Treatment of shock with vasodilators measuring skin temperature on the big toe. Diseases of the Chest, 52, 425.

Pagliero, K. M., Yates, A. K., and Hill, D. G. (1973). The controlled use of vasodilator drugs in the management of hypovolaemia complicated by diminished cardiac function. British fournal of Surgery, 60, 201.

Ross, B. A., Brock, R., and Aynsley-Green, A. (1969). Observations on central and peripheral temperatures in the understanding and management of shock. British fournal of Surgery, 56, 877.

Spitzer, A. G., and Brock, R. (1968). The recognition of hypovolaemia after open heart surgery. Guy's Hospital Reports, 117, 131.

Correspondence to Dr. A. Aynsley-Green, Department of Paediatrics, The John Radcliffe Hospital, Headington, Oxford OX3 9DU.

\section{Nigerian Journal of Paediatrics}

The Archives has long had links with Nigeria, for we have published many articles from that country. We are glad to welcome this new venture, The Nigerian Journal of Paediatrics, which started publication at the beginning of 1974. An additional link is provided by the presence on its Editorial Committee of a former Editor of the Archives, Professor R. H. Dobbs. 